\title{
Fast Molecular-Based Detection of SARS-CoV-2 Virus from Saliva by Direct RT-PCR
}

\author{
François DUBOURNAIS*, Cécile MAES \\ Molecular biology section, CERES Laboratory, ACMARIS S.A.S.
}

*Corresponding Authors: François DUBOURNAIS, Molecular biology section, CERES Laboratory, ACMARIS S.A.S.

\begin{abstract}
Since the COVID-19 occurs in Wuhan, because of the novel coronavirus $n$ Cov-2019, that was soon named SARS-Cov-2 due to closed genomic homology and clinical symptoms with SARS-Cov, authorities and the scientific community have emphasized the necessity of a mass screening campaign in order to control and prevent clusters and the epidemic from progressing. Indeed, the SARS-Cov-2 virus has a higher reproductive rate $(R O)$ than $S A R S-C o V$ and MERS-CoV. This infection may mobilize many health professionals, and may present high short and/or long-term health risk. It is obvious that any reliable, simple, painless and sensitive diagnostic systems are needed to control the pandemic. Yet, very few sample and analytic methods are recognized as reference points, i.e., nasopharyngeal swabs (NPS) and detection of viral RNA by real-time reverse transcription polymerase chain reaction ( $r R T-P C R)$. To avoid painful sampling, testing hesitancy, healthcare professional contamination, and to access fast and reliable analytic methods, existing adjustments to testing methods are needed to be evaluated. Posterior oropharyngeal saliva is a sensitive source for SARS-CoV-2 detection. Stability of SARS-CoV-2 detection in simple saliva tube sampling without supplementation has been demonstrated, following RNA extraction and purification, or by direct rRT-PCR. The aim of this study is to investigate the feasibility of saliva direct rRT-PCR molecular diagnosis, comparing to NPS direct rRT-PCR, and a limited limit of detection determination to evaluate performance. It provides a much-needed alternative at exactly the right time.
\end{abstract}

Keywords: SARS-CoV-2 - Saliva - RT-PCR - threshold level - diagnosis

\section{INTRODUCTION}

SARS-CoV-2 airborne virus generates the COVID-19 disease, especially Severe Acute Respiratory Syndrome (SARS). Many countries are practicing population-wide testing strategy in order to rapidly decrease the spread of the disease, to reduce pressure on the healthcare system, to provide care, or even to eliminate the disease altogether [1].

Many human fluids have been investigated to determine the most relevant moment in time and the best site for sampling occur. Indeed, in a broad pandemic context, such as it is, indeed other contexts, the least painful, fastest and most secure the sampling is, the easier mass screening will be.

As a respiratory infection, despite the broad and non-specific case definitions, nasopharyngeal swabs (NPS) sampling appeared first as the gold standard method for SARS-CoV-2 diagnosis by viral RNA amplification rRT-PCR, due to its sensitivity, reliable performance, and the viral load in the upper respiratory tract $[2,3]$. NPS seems to take a longer time than other sampling sites for RNA positive detection after disease onset [3], probably due to the presence of residual viral particles, while saliva loads are highest during the major phase of contagiousness (about one to 2 weeks). Otherwise, as salivary droplets represent the main source of human-to-human transmission [4], the angiotensin converting enzyme 2 (ACE2), the receptor for SARS-CoV-2 entry in the cell, are highly expressed in the oral cavity. Thus, it is rational to use saliva as a diagnostic substrate for SARS-CoV-2 infection. It has been proved that COVID-19 diagnosis by NPS and saliva have a high rate of success [5]. Moreover, saliva-based direct rRT-PCR testing has been studied, focusing on RNA ORF1ab and N gene viral sequencing, among others [6], so that it was interesting to extend this research to another commercial ORF1ab and $\mathrm{N}$ gene targets using kit.

NPS can simply cause discomfort, but it can also cause nose bleed, scarring tissue, personal healthcare risks, and sampling may be impossible for physical or pathological reasons. Thus, repeating the same sampling strategy may be defeated. 
Combining mass screening, i.e., less restrictive sampling methods when possible, and a rapid reference method of analysis is key. Older and recent studies have shown saliva as point-of-care matrix in early diagnosis by molecular assay $[7,8,9,20]$.

Saliva contains a wide range of components, such as pro-inflammatory cytokines, chemokines, matrix metalloproteinases, mitochondrial DNA, genomic DNA, bacteria, SARS-CoV and SARS-CoV-2 virus, SARS-CoV antibodies, miRNA and extracellular vesicles, numerous defence proteins and peptides [10], that may potentially interfere with RT-PCR amplification.

The French High Health Authority (HAS) gave 2020 a favourable opinion on the use of saliva samples in a specific context as a specimen for SARS-CoV-2 detection and diagnosis in September [11]. However, HAS specified that the cycle threshold $(\mathrm{Ct})$ used to differentiate a positive from a negative case, which should be between 35 and 40 .

In order to find a way of combining an alternative sampling method made of saliva, with a rapid reference diagnostic response by using direct rRT-PCR without extraction, the aim of this study was to investigate posterior oropharyngeal saliva samples of asymptomatic cohort volunteers' effects on rRT-PCR results, including sample enrichment with SARS-CoV-2 wild sources, and a limited determination of limit detection in saliva template by spiking viral gold-standards to evaluate performance.

\section{MATERiAls AND MethodS}

\subsection{Study Frame}

This study was conducted at CERES ACMARIS S.A.S Laboratory in Villeneuve de Berg (France) with a cohort volunteers without signs / symptoms, after signing a consent form. 15 male and female persons between 25 and 45 years old had their saliva samples tested, having confidentially been asked about their medical history. No sample was found to be SARS-CoV-2 positive.

To validate saliva as biological sample for SARS-CoV-2 diagnosis, Saliva and Saliva pooled samples were tested in order to evaluate Rnase P mRNA target as RT-PCR and sufficient cellular material control.

Apart from the use of delta $\mathrm{Ct}$ for performance evaluation, quantification was done using the gold standard inactivated calibration curve.

\subsection{Sample Collection}

Saliva was collected in $15 \mathrm{~mL}$ NEST centrifuge PP sterile RNase/DNase free tubes, containing previously $1 \mathrm{~mL}$ VTM transport medium (Capricorn Scientific GmbH VTM-49 CE-IVD). Volunteers were asked not to eat or drink at least half an hour before sampling took place, then to completely swallow saliva and bring up saliva from the back of throat, exhaling and clearing their throat, then to spit at least $1 \mathrm{~mL}$ into the tube [8] [9]. Samples were stored immediately at $5+/-3{ }^{\circ} \mathrm{C}$ until they were tested. Volunteers collected the samples themselves under team supervision, according to the procedure shown in Fig 1.

The term Saliva in this study means Saliva:VTM (50:50 - vol:vol).

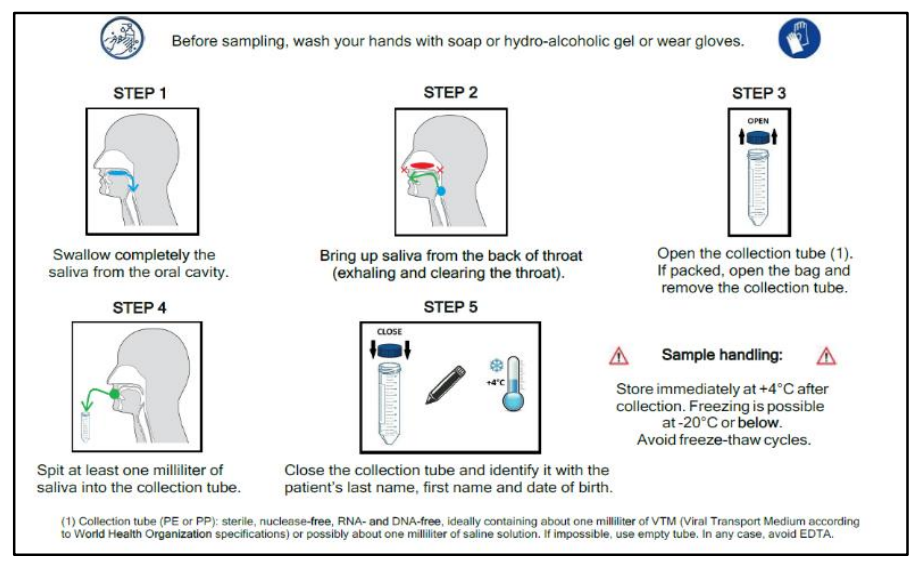

Fig1. Saliva sampling procedure - instructions for use. See annexe A. 


\subsection{Viral Load}

Different viral loads were achieved by serial dilutions of high viral loads outpatients NPS, i.e., wild sources. Based on Rnase P Ct determination, various amounts of homogenised and characterized NPS samples were used to load freshly-treated constant saliva templates as shown in Fig 4. Except for the highest load (NPS solution/saliva - 2:9 - vol:vol), the amounts of NPS never exceeded 3\% of the saliva template (vol:vol).

\subsection{Limit of Detection (LOD)}

Considering the dynamics of SARS-CoV-2 in the saliva of infected patients with mild or severe COVID-19, the viral load seems to peak during the first week of symptoms (about $10^{5}$ to $10^{7}$ copies $/ \mathrm{mL}$ ) then to decline to $10^{3}$ copies $/ \mathrm{mL}$ up to 21 days after symptom onset $[12,13]$. Otherwise, the range of viral load in hospitalized patients can change between $9,9 \times 10^{2}$ and $1,2 \times 10^{8}$ copies $/ \mathrm{mL}$ [8]. Therefore, it was of immediate interest to evaluate a LOD below $9,9 \times 10^{2}$ copies $/ \mathrm{mL}$. The limit of detection for SARS-CoV-2 was tested at a concentration of 500 copies $/ \mathrm{mL}$ using SARS-CoV-2 gold standard (Qnostics SARS-CoV-2 Q control 01 SCV2QC01-A) into saliva and saliva pooled specimens.

\subsection{Integrative Standard Calibration for Quantification}

In order to guide the loads, pick the gold standard for LOD assessment and test pre-treated saliva rRTPCR, a Pearson's correlation was made, based on $\mathrm{N}$ gene target $\mathrm{Ct}$ (Crossing threshold). As a challenge, we investigated correlation using SARS-CoV-2 gold standard in different conditions (VTM, molecular biology quality water, lysis buffer named sample release reagent SANSURE Biotech). Serial dilutions were made in these solutions of 10000, 7500, 5000, 2500, 1250, 625, 312, 156, 78 and 39 copies / $\mathrm{mL}$, then tested in triplicate each. Pearson's correlation and significance level are shown in Fig 2.

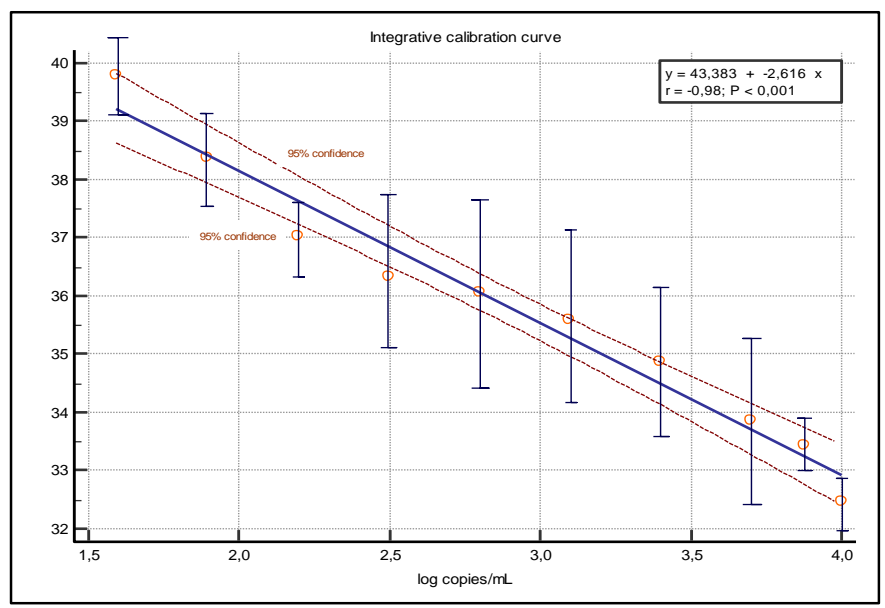

Fig2. Pearson's correlation between $N$ gene Ct (Crossing threshold of $N$ gene) and SARS-CoV-2 copies $/ m L$. The correlation coefficient was 0.98 ( $P<0.0001,95 \%$ CI -0,9963 to -0,9308), PCR efficiency $140 \%$.

\subsection{SARS-CoV-2 RNA Detection}

In order to inactivate virus and partially neutralize or reduce inhibitors in crude saliva, we tested an approach that has been demonstrated to improve viral RNA detection within a pre diluted saliva material $[6,19]$. We avoid voluntarily EDTA supplementation due to our experiment in order to reduce background fluorescence noise. After being brought to room temperature, samples were heated at $65^{\circ} \mathrm{C}$ for 20 minutes, then vortexed at maximal speed for 15 seconds to disrupt mucous blocks.

The SARS-CoV-2 N gene and ORF1ab targets detection were achieved on CFX96 Touch deep well real-time PCR detection system (Bio-Rad) using Novel Coronavirus (2019-ncoV) Sansure Biotech reagents.

An aliquot of each sample (at least $250 \mu \mathrm{L}$ ) was transferred to a $1,5 \mathrm{~mL}$ molecular biology quality microtube, and mixed with an equivalent volume of lysis buffer (Sample Release Reagent provided by Sansure Biotech). After incubation at room temperature for at least 10 minutes, vertexing and short spin centrifugation, RT-PCR was performed, according to the manufacturer's specifications. 
Amplification of the genomic target regions ORF1ab and $\mathrm{N}$ gene were simultaneously done with Rnase P amplification as PCR and cellular matrix control to validate each reaction.

Data were managed with Bio-Rad CFX Manager Industrial Diagnostic Edition 3.0 V 4.1.2433.1219, with settings on baseline subtracted curve fit and baseline auto calculated. Positives and negatives controls were done for each run.

\subsection{Characterisation of SARS-CoV-2 Wild Sources}

Of interest, wild viral sources were needed to compare rRT-PCR response, behaviour and performance between the two methods (NPS direct rRT-PCR versus Saliva direct rRT-PCR).

Thus, the selection of outpatient' samples was key. We estimated that these samples may have a linear relationship within serial dilutions, at least for Rnase $\mathrm{P}$ control and one of the viral target sequences.

Two samples were picked for absence of visual mucous aggregates, high viral load to achieve dilution in an interpretable manner, and good response of the targets even for ORF1ab, despite significant linear regression for the latter (results are shown in Fig. 3).

\subsection{Statistical Analysis}

We did statistical analyses with MedCalc® Statistical Software version 19.6.4 (MedCalc Software Ltd, Ostend, Belgium).

The SARS-CoV-2 rRT-PCR Sansure Biotech on NPS admitted and the French National Reference Centre validated method were considered as reference method in this study. Assuming results comparison between different sample specimens and different pre-analytic phase when it comes to methods of comparison, we used Passing and Bablok regression, providing proper conclusions such as linear relationship, bias, proportional differences, and allowing correction actions if needed to draw positive / negative threshold $[14,15]$.

\section{Results}

Between Janv 02, 2021 and Janv 31, 2021, about 10 wild SARS-CoV-2 from patient's NPS materials were characterized using rRT-PCR Sansure Biotech reagents and diluted in Sample Realease Reagent (SRR) provided by the manufacturer. Of these, two candidates (NPS1 and NPS3) were approved as good templates for investigation.

Interestingly, except for Rnase P, overall standard deviation (SD) of triplicates was higher at highest and lowest viral loads for ORF1ab and $\mathrm{N}$ gene targets (for instance: $\mathrm{N}$ gene NPS1 undiluted SD+/1.77 Ct, N gene NPS3 undiluted SD+/- 1.81 Ct / ORF1ab NPS3 1:10000 SD+/- 3.82 Ct).

Acceptable correlation was observed for Rnase $\mathrm{P}$ target within serial dilutions, for $\mathrm{N}$ gene, but not for ORF1ab, despite visual coherency for the latter (Fig 3).

Compared rRT-PCR responses between NPS diluted in VTM and in Saliva (Saliva:VTM, 50:50, vol:vol), were investigated.

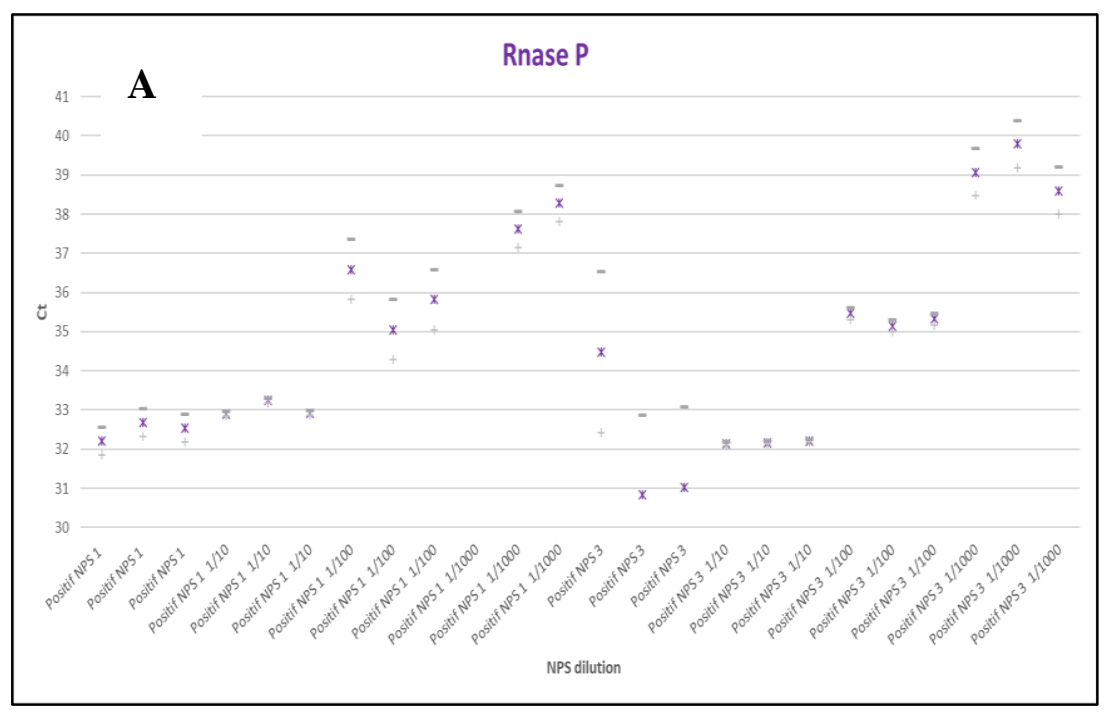



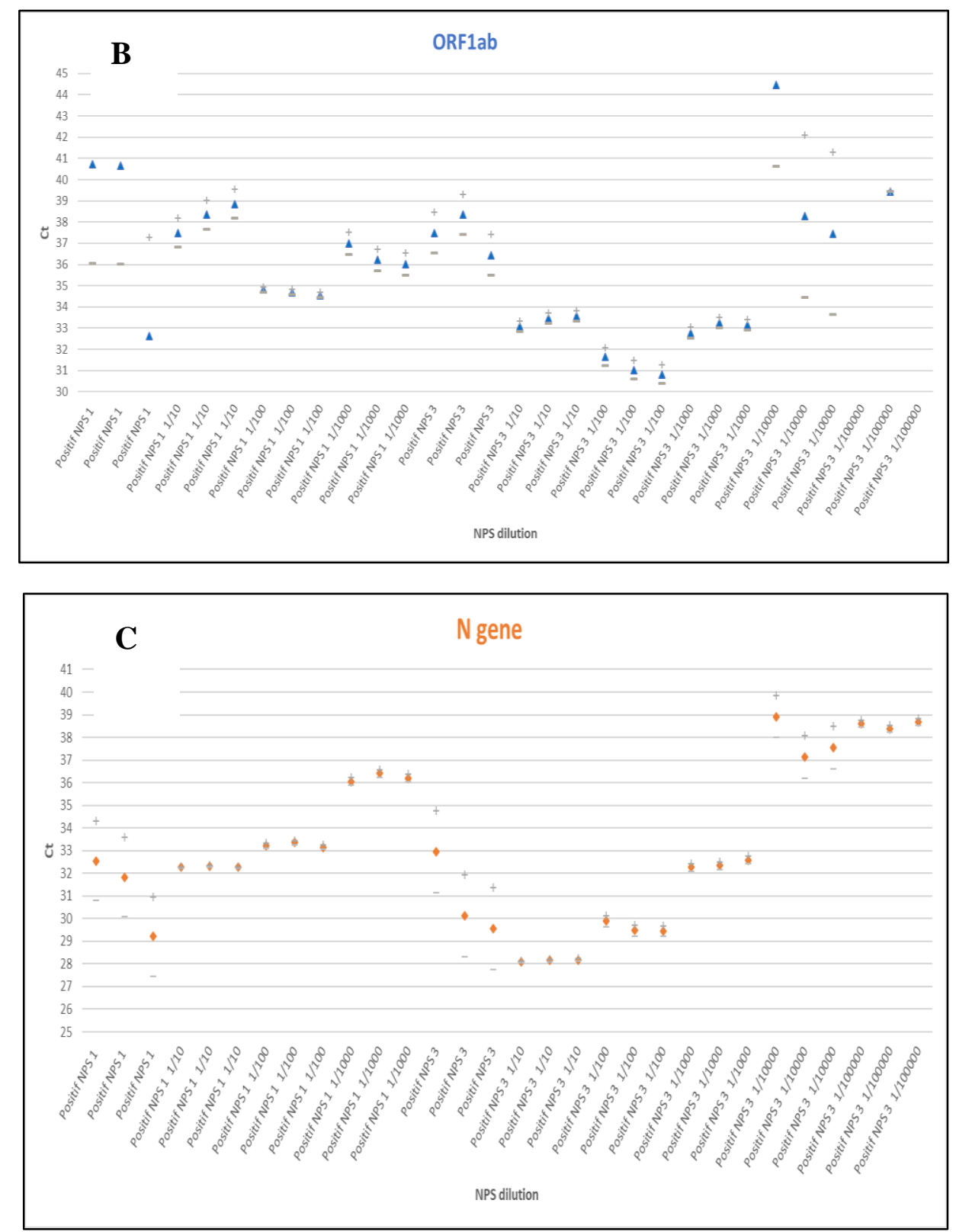

Fig3. NPS samples numberl and 3 rRT-PCR characterization in triplicates per dilution. + and - represent SD.

A: Rnase P target Ct - dilutions $1 / 10000$ \& 1/100000 not detected (not shown) - For NPS 1 and 3 respectively, the correlation coefficient was $-0.95(P<0.0001,95 \%$ CI -0.9876 to -0.8181$)$ and $-0.90(P=0.0001,95 \% C I-$ 0.9719 to -0.6743$)$.

B: ORFlab target Ct - low linear relationship for both NPS.

$C: N$ gene target $C t-$ For NPS 1 and 3 respectively, the correlation coefficient was $-0.89(P=0.0001,95 \% C I-$ 0.9699 to -0.6552$)$ and $-0.71(P=0.0144,95 \%$ CI -0.9186 to -0.1918$)$.

Based on N gene target quantification, initial NPS viral load was estimated for NPS1 and NPS3 at $2.3 \times 10^{6}$ and $3.0 \times 10^{6}$ copies / $\mathrm{mL}$ respectively.

For Saliva testing, the same amount of Saliva was used, based on Rnase P Ct control (results are shown in Fig. 4).

Assuming that no measurement should be excluded from the data, without making assumptions about the distribution of results, a Passing \& Bablok regression was attempted (Fig 5). With level of significant threshold $\alpha=0,05$ (confident interval of 95\%), cusum tests indicated no significant deviation from linearity (ORF1ab and $\mathrm{N}$ gene $\mathrm{p}$ value 0.53 and 0.80 , respectively). A visual linear relationship is accessible, as allowed Passing \& Bablok graphs in Fig 5. Interestingly for $\mathrm{N}$ gene, intercept included value zero and slope value one. The same for ORF1ab about the slope, so it can be 
concluded that there was no significant difference between the two methods for $\mathrm{N}$ gene target, and no proportional difference for ORF1ab target.
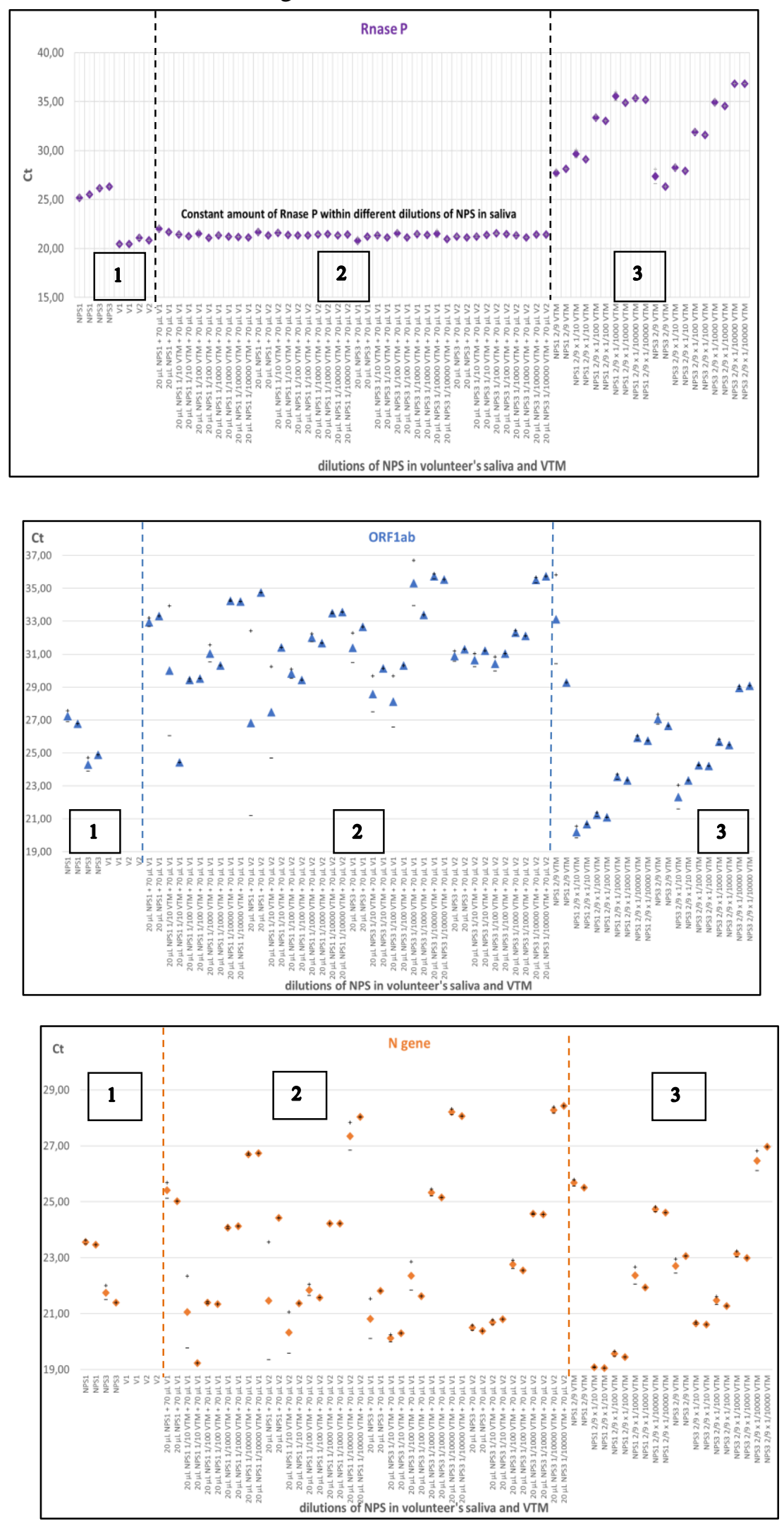

Fig4. $r R T-P C R$ Ct results in duplicate each, + and-representing $S D$,

Parts 1 : NPS (1 and 3), volunteer's Saliva (V1 and V2) that were negatives for viral targets,

Parts 2 : different dilutions of NPS in volunteer's Saliva (V1 and V2) with the same amount of Saliva template based on Rnase P Ct (NPS1 + V1, NPS1 + V2, NPS3 + V1, NPS3 + V2),

Parts 3 : NPS with the same viral loads than in dilutions with Saliva, where Saliva were replaced by VTM. 
However, the ORF1ab target exhibited an obvious constant difference (bias) between the two methods. Moreover, one point was identified to be an outlier, i.e., residual in the Passing \& Bablok regression. Since the procedure assumed a linear relationship, which was the case, we verified the distribution and founded that ORF1ab target was closed to a normal distribution (Shapiro-Wilk test at $\alpha=0.050, p$ value $=0.399)$, and the observed residual showed a random pattern.

Compared to NPS1/NPS3 VTM patterns, Saliva dilutions of NPS1 and NPS3 showed surprisingly very closed or lowest SD, suggesting low or no accuracy variation in Saliva vs VTM.
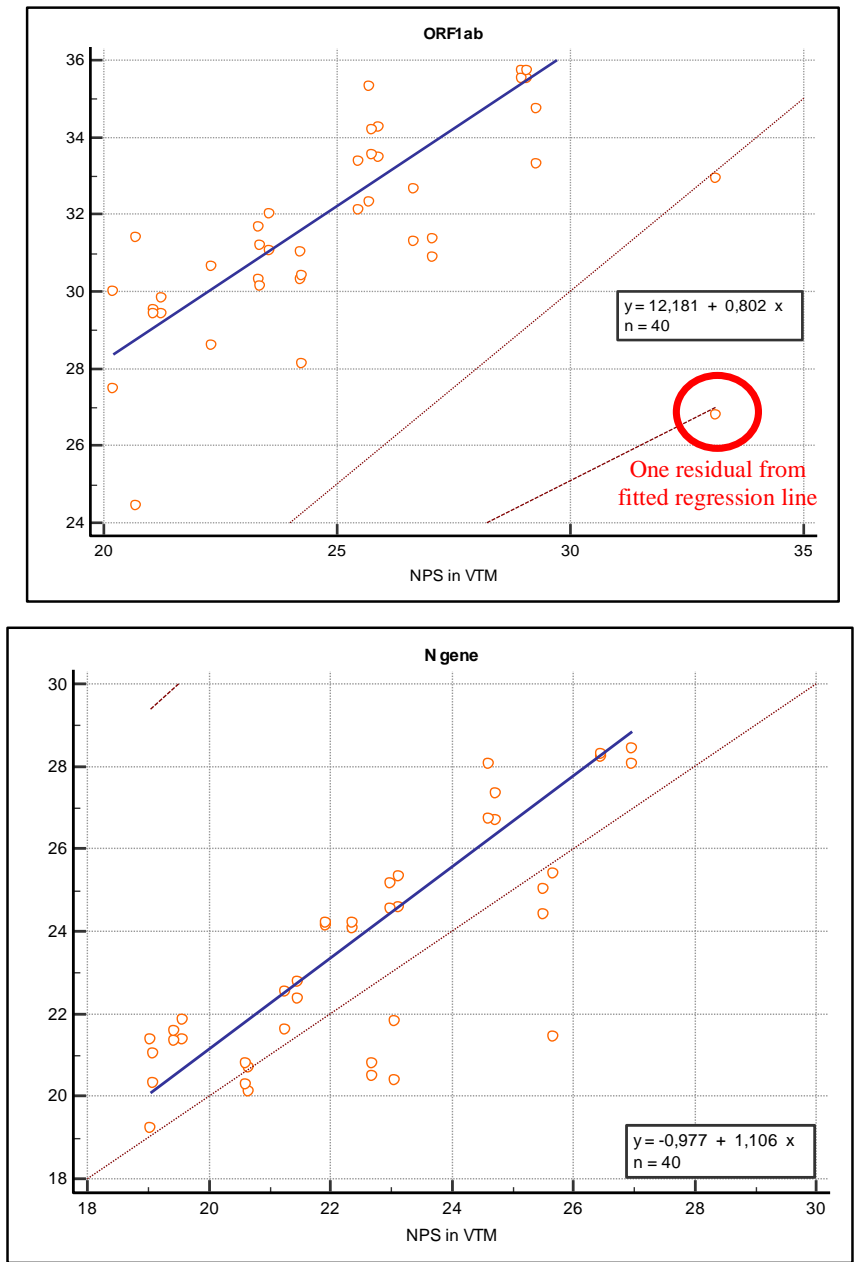

\begin{tabular}{|c|c|c|c|c|}
\hline \multirow{2}{*}{ Sample size / type } & \multicolumn{2}{|c|}{$40 \times$ ORF1ab } & \multicolumn{2}{|c|}{$40 \times \mathrm{N}$ gene } \\
\hline & NPS in VTM & NPS in Saliva & NPS in VTM & NPS in Saliva \\
\hline Lowest value & 20,19 & 24,43 & 19,05 & 19,23 \\
\hline Highest value & 33,12 & 35,74 & 26,96 & 28,42 \\
\hline Arithmetic mean & 25,06 & 31,55 & 22,56 & 23,43 \\
\hline Median & 24,86 & 31,34 & 22,53 & 22,66 \\
\hline Standard deviation & 3,30 & 2,59 & 2,44 & 2,74 \\
\hline Standard error of the mean & 0,52 & 0,41 & 0,39 & 0,43 \\
\hline Regression Equation & \multicolumn{2}{|c|}{$y=12,18+0,80 x$} & \multicolumn{2}{|c|}{$y=-0,98+1,11 x$} \\
\hline \multicolumn{5}{|l|}{ Systematic differences } \\
\hline Intercept A & \multicolumn{2}{|c|}{12,18} & \multicolumn{2}{|c|}{$-0,98$} \\
\hline $95 \% \mathrm{CI}$ & \multicolumn{2}{|c|}{6,71 to 16,59} & \multicolumn{2}{|c|}{$-7,67$ to 2,74} \\
\hline \multicolumn{5}{|l|}{ Proportional differences } \\
\hline Slope B & \multicolumn{2}{|c|}{0,80} & \multicolumn{2}{|c|}{1,11} \\
\hline $95 \% \mathrm{CI}$ & \multicolumn{2}{|c|}{0,61 to 1,00} & \multicolumn{2}{|c|}{0,95 to 1,40} \\
\hline \multicolumn{5}{|l|}{ Random differences } \\
\hline Residual Standard Deviation (RSD) & \multicolumn{2}{|c|}{2,06} & \multicolumn{2}{|c|}{1,22} \\
\hline \pm 1.96 RSD Interval & \multicolumn{2}{|c|}{$-4,04$ to 4,05} & \multicolumn{2}{|c|}{$-2,38$ to 2,39} \\
\hline \multicolumn{5}{|l|}{ Linear model validity } \\
\hline Cusum test for linearity & \multicolumn{2}{|c|}{$(\mathrm{P}=0,53)$} & \multicolumn{2}{|c|}{$(\mathrm{P}=0,80)$} \\
\hline
\end{tabular}

Fig5. Passing and Bablok regression between NPS in water against NPS in Saliva for ORFlab and N gene targets. Ct are represented and analysed. Theorical line is dotted. 
Therefore, overall Passing \& Bablok correlation tests confirmed a high agreement between the two matrices (VTM vs Saliva) for N gene target $(\alpha=0.05$; $\mathrm{p}$ value $=0.80$ ), and bias except, for ORF1ab target $(\alpha=0.05 ; \mathrm{p}$ value $=0.53)$. Observed bias for ORF1ab will be used for level threshold determination.

Assessment of the LOD from 5000 to 500 viral copies $/ \mathrm{mL}$ clearly showed a broad Standard Deviation (Fig. 6). Approaching the LOD, we considered this deviation as logic, accuracy decreasing dramatically in these circumstances. However, except uncommon non detected $\mathrm{Ct}$ (no response of the target), the profiles in triplicates showed good and clear $\mathrm{S}$ curves, even at 500 copies $/ \mathrm{mL}$, in all Saliva patterns. Despite reliability decreasing and regardless of the $\mathrm{Ct}$, targets detection was achieved at a maximum experimental rate in saliva patterns around 500 copies $/ \mathrm{mL}$ (see table $1-625$ and 312 copies/mL).

Using RNA purification / concentration, the best NPS LOD value appears to be 1000 copies/mL [16], and about 5000 copies $/ \mathrm{mL}$ in Saliva [17]. In order to evaluate the robustness of the direct Saliva rRTPCR method and achieve optimal performance of detection, considering the results in Fig. 6, the LOD of 500 copies $/ \mathrm{mL}$ was picked to be tested in 26 independent repeats (26 independent Saliva patterns made of individual or pooled Saliva), in quadruplet each (see Fig.7).

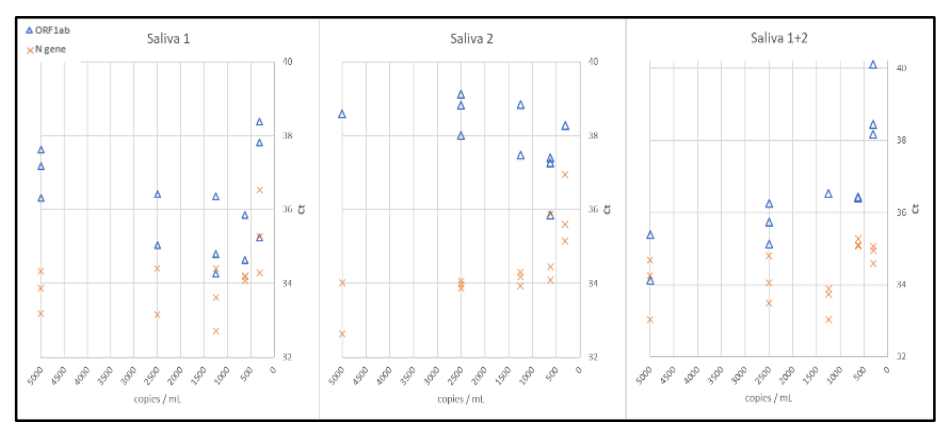

Fig6. Assessment of the limit of detection (LOD). Inconclusive or non-detected values were excluded. Different amounts of SARS-CoV-2 gold standard in 3 saliva patterns (V1,V2, V1 + V2), in triplicate each, from 5000 to 500 copies $/ \mathrm{ml}$.

Table1. Detection rate in Saliva for LOD assessment.

\begin{tabular}{|l|c|c|c|c|c|}
\hline Sample type (copies/mL) & 5000 & 2500 & 1250 & 625 & 312 \\
\hline ORF1 ab & $6 / 9$ & $8 / 9$ & $6 / 9$ & $7 / 9$ & $7 / 9$ \\
\hline N gene & $8 / 9$ & $8 / 9$ & $9 / 9$ & $9 / 9$ & $9 / 9$ \\
\hline
\end{tabular}

Remarkably, Rnase P was clearly detected between 20 to $28 \mathrm{Ct}$ with a $100 \%$ rate, in accordance with the benefit of validating and verifying human cellular sufficient matter and PCR control. Underlying a good rate of $\mathrm{N}$ gene detection (73\%), ORF1ab exhibited a worse rate that initially seemed unlikely $(15 \%)$.

Based on Rnase $\mathrm{P}$ and $\mathrm{N}$ gene sequences amplification rate, we assumed that a higher LOD should be tested, around 1000 copies/mL to obtain a better rate for ORF1ab, but performances seemed to show an acceptable LOD in a diagnostic way, considering the viral loads frequently found in Saliva in the early stage of infection, from 1 to 2 weeks after symptom onset, and even went up to 20 days in accordance with certain studies.

\section{DISCUSSION}

Our findings demonstrated Ct shifts may or may not take within Saliva against NPS pattern on direct rRT-PCR, in order to help and lead biologists towards an informed diagnosis. Except in extreme conditions that can be detected thanks to internal control Rnase P, linear relationship and good agreement have been found in the range of 18 to $30 \mathrm{Ct}$ between the two methods for Rnase $\mathrm{P}, \mathrm{N}$ gene and ORF1ab targeting, with a bias shift for ORF1ab. In order to avoid false negative results, i.e., low reliability around 500 copies $/ \mathrm{mL}$ of viral load and potential false positive results due to sampling contamination, considering ORF1ab response variability in NPS routine testing, and a real bias shift in Saliva compared to NPS within the range 20 to $30 \mathrm{Ct}$ and probably for highest $\mathrm{Ct}$, we underline the necessity to set a positive / negative threshold value at $35 \mathrm{Ct}$ for ORF1ab target.However, taking into account no obvious shift of $\mathrm{N}$ gene $\mathrm{Ct}$ response between NPS and Saliva templates, and an acceptable rate of detection around 500 copies $/ \mathrm{mL}(73 \%)$, the positive / negative threshold for diagnostic 
decision should be set at the median that has been observed for LOD testing (37,5 Ct), corresponding otherwise between low and at times very low, but positive results provided by the French Microbiology Society to the French Ministry of Health September 11, 2020

[18].

Besides methods comparison studies, we underline the necessity of rRT-PCR testing with wild sources, taking into account multiple mutation evolution, regardless of the amplification targets.

In this study, we achieved and demonstrated SARS-coV-2 RNA detection in multiple Saliva patterns using Sansure Biotech reagents, direct rRT-PCR and a modified pre analytic procedure. As a guideline, we recommend the practice of such a diagnosis in the early stage of infectious or symptomatic patients, and if NPS is impossible, due to some patient' health conditions, according to HAS specifications. Nevertheless, we demonstrated the feasibility of low viral loads detection, accuracy and de facto reliability decreasing dramatically to around the limit of detection that we estimated between 500 and 1000 viral copies $/ \mathrm{mL}$.

Even with a limit of detection that can guarantee a low viral load detection (about $1000 \mathrm{copies} / \mathrm{mL}$ ), saliva is a very complex specimen that can inhibit or disturb performances of the method. Indeed, we founded empirically that some medical treatments would probably lead to global rRT-PCR inhibition. These cases were founded to inhibit all tests we realized. Medical treatments in question were: formoterol fumarate and budesonide (aerosol taken the day before sampling), amphotericine B with sulfites and PARABEN (mouth rinse realized the day on the evening before sampling),

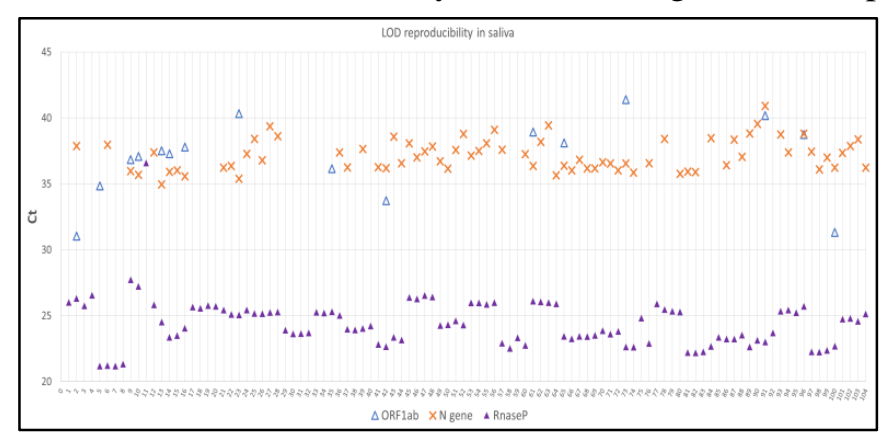

Fig7. LOD reproducibility assessment in 26 independent repeats and Saliva patterns (Saliva and pooled Saliva templates at different days). Rnase P target exhibited $100 \%$ response, $N$ gene $73.1 \%$ and ORF lab only $15.4 \%$.

beclomethasone (aerosol taken the day on the evening before sampling). These pharmaceuticals are respectively bronchodilator (persisting action at least 12 hours in the lung), glucocorticosteroid, antifungal and steroid. Formoterol fumarate, budesonide and beclomethasone were found to be taken by inhalation, with the assumption it persists in the respiratory tracts and present in oropharyngeal Saliva.

Direct rRT-PCR on NPS and Saliva specimens, according to specific sampling and conservative conditions, in association with a safety and quick pre analytic modes, allow fast and reliable SARSCoV-2 diagnosis, avoiding false negative and positive results with Rnase $\mathrm{P}$ as internal control, and ORF1ab, $\mathrm{N}$ gene $\mathrm{Ct}$ decision threshold respect.

Excluding some special and uncommon patterns, Saliva may be used as a choice specimen for most patients who have difficulty with the NPS method, in logistical constraint conditions, even for children, to achieve mass screening efficiency.

We highlight some questions, for instance due to prolonged supine position. In these cases, exhaling and clearing the throat for sampling may be difficult. On the other hand, a small size of sample may suffice, due to bronchopulmonary secretions moving by ciliary activity to the posterior oropharyngeal area. Therefore, Saliva may be used for bedridden patients.

Early detection and isolation of cases is strategic for outbreak control. Viral load monitoring, i.e., repeated sampling, may help to understand treatment strategy, to control isolation duration, to investigate disease evolution case by case when needed. Without replacing NPS, Saliva sampling is an alternative and efficient tool. Currently, there are many available studies dealing with the role of Saliva in the detection of SARS-CoV-2. Our research has some limitations and should be follow-up with a LOD testing at 1000 copies $/ \mathrm{mL}$, with a broader panel of Saliva donors to investigate and 
identify a better range of limitations, e.g., pharmaceutical interactions, and, despite high loads frequently found in Saliva, in the range between 30 and $40 \mathrm{Ct}$ to better amplify our understanding of behaviour.

\section{ACKNOWLEDGMENTS}

We gratefully acknowledge all our CERES/ACMARIS colleagues fighting daily to do the best. Special thanks to Natacha Chamourin and Alexis Duquesnoy for their contribution.

\section{REFERENCES}

[1] European Centre for Disease Prevention and Control, Population-wide testing of SARS-CoV-2 : country experiences and potential approaches in the EU/EEA and the United Kingdom. (2020).

[2] Wang W., Xu Y., Gao R., Detection of SARS-CoV-2 in Different Types of Clinical Specimens, JAMA. 323 (18).

[3] Wang H., Liu Q., Hu J. et al., Nasopharyngeal Swabs Are More Sensitive Than Oropharyngeal Swabs for COVID-19 Diagnosis and Monitoring the SARS-CoV-2 Load, Front. Med.. 7 (334) (2020).

[4] Han P., Ivanovski S., Saliva - Friend and Foe in the COVID-19 Outbreak, Diagnostics. 10(5) (290) (2020).

[5] Nunes Vaz S., Souza de Santana D., Martins Netto E. et al., Saliva is a reliable, non-invasive specimen for SARS-CoV-2 detection, Braz. J. Infect. Dis. 24(5) (2020).

[6] Ranoa D.R., Holland R., Fadi G. et al., Saliva-Based Molecular Testing for SARS-CoV-2 that Bypasses RNA Extraction, 10.1101/2020.06.18.159434

[7] Azzi L., Carcano G., Gianfagna F. et al., Saliva is a reliable tool to detect SARS-CoV-2, J. Infect. 81(1) (2020).

[8] To K.K., Tsang O.T., Yip C.C. et al., Consistent Detection of 2019 Novel Coronavirus in Saliva, Clin. Infect. Dis. 71(15) (2020).

[9] Azzi L., Maurino V., Baj A. et al., Diagnostic Salivary Tests for SARS-CoV-2, J. Dent. Res. 100(2) (2021).

[10]Fábián T.K., Hermann P., Beck A. et al. Salivary Defense Proteins: Their Network and Role in Innate and Acquired Oral Immunity, Int. J. Mol. Sci. 13(4) (2012).

[11] French High Health Authority, Opinion $\mathrm{n}^{\circ} 2020.0047 / \mathrm{AC} / \mathrm{SEAP}$ on the inclusion in the list of documents and benefits referred to in article L. 162-1-7 of the Code la Sécurite Sociale, the detection of the virus SARS-CoV-2 genome by reverse transcription followed by salivary sample amplification (RT-PCR). (2020).

[12]To K. K., Tsang O.T., Leung W.-S. et al., Temporal profiles of viral load in posterior oropharyngeal saliva samples and serum antibody responses during infection by SARS-CoV-2 : an observational cohort study, Lancet Infect. Dis. 20(5) (2020).

[13]Zhu J., Guo J., Xu Y. et al., Viral dynamics of SARS-CoV-2 in saliva from infected patients, J. Infect. 81(3) (2020).

[14] Bilić-Zulle L., Comparison of methods : Passing and Bablok regression, Biochem. Med. 21(1) (2011).

[15] Passing H., Hoechst A.G., Bablok W. et al., A New Biometrical Procedure for Testing the Equality of Measurements from Two Different Analytical Methods, J. Clin. Chem. Clin. Biochem. 21(11) (1983).

[16] Wyllie A.L., Fournier J., Casanovas-Massana A., Saliva is more sensitive for SARS-CoV-2 detection in COVID-19 patients than nasopharyngeal swabs, medRxiv, [Pre-print]. (2020).

[17] Wyllie A.L., Fournier J., Casanovas-Massana A., Saliva or Nasopharyngeal Swab Specimens for Detection of SARS-CoV-2, N. Engl. J. Med. 383(13) (2020).

[18] French Microbiology Society (SFM), Opinion on the interpretation of the value of Ct (estimation of viral load) obtained in case of positive RT-PCR SARS-CoV-2 on clinical samples carried out for diagnostic or screening purposes, Electronic Concordance Update, to the request of the French General Direction of Health. Version 3 _ 07/10/2020.

[19]Lalli M.A., Chen X., Langmade S.J. et al. Rapid and extraction-free detection of SARS-CoV-2 from saliva with colorimetric LAMP, medRxiv [Pre-print]. (2020).

[20] Iwasaki S., Fujisawa S., Nakakubo S. et al., Comparison of SARS-CoV-2 detection in nasopharyngeal swab and saliva, J. Infect. 81 (2020). 


\section{Annex A \\ SALIVA SAMPLING PROCEDURE SARS-COV-2 RT-PCR Test}

All saliva collected corresponds to a standard method for some biological tests, especially for the detection of SARS-Cov-2 virus. The tubes are for single use.

Instructions for use:

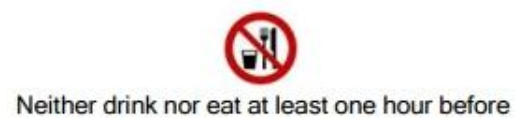

Before sampling, wash your hands with soap or hydro-alcoholic gel or wear gloves.

STEP 1

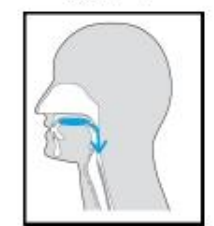

Swallow completely the saliva from the oral cavity.

STEP 4

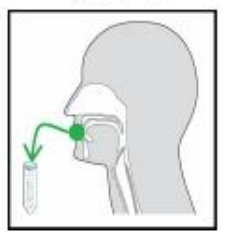

Spit at least one milliliter of saliva into the collection tube.
STEP 2

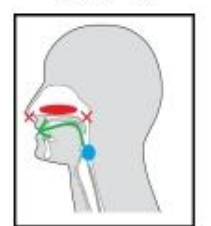

Bring up saliva from the back of throat (exhaling and clearing the throat).

STEP 5

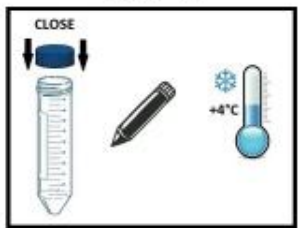

Close the collection tube and identify it with the patient's last name, first name and date of birth.
STEP 3

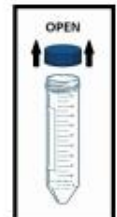

Open the collection tube (1) If packed, open the bag and remove the collection tube.

\section{$\triangle$ Sample handling: $\triangle$}

Store immediately at $+4^{\circ} \mathrm{C}$ after collection. Freezing is possible at $-20^{\circ} \mathrm{C}$ or below. Avoid freeze-thaw cycles. (1) Collection tube (PE or PP): sterile, nuclease-free, RNA- and DNA-free, ideally containing about one millititer of VTM (Viral Transport Medium according
to World Health Organization specifications) or possibly about one milliliter of saline solution. If impossible, use empty tube. In any case, avoid EDTA.

\section{If steps 1 to 4 are unachievable, proceed as follow:}

Depending on the patient's health, sampling is possible (from a third person) using a sterile pipette.

For children younger than 6 years of age, plan for the presence and supervision of an adult.

\section{STERILE PIPETTE USING}

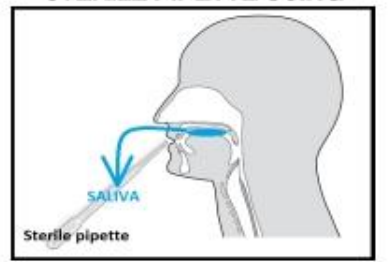

After collection, put the content of the pipette into collection tube then proceed to STEP 5 .

Citation: François DUBOURNAIS \& Cécile MAES, " Fast Molecular-Based Detection of SARS-CoV-2 Virus from Saliva by Direct RT-PCR ", International Journal of Clinical Chemistry and Laboratory Medicine (IJCCLM), 7(1), pp.1-11, 2021. Available: DOI: http://dx.doi.org/10.20431/2455-7153.0701001

Copyright: (C) 2021 Authors. This is an open-access article distributed under the terms of the Creative Commons Attribution License, which permits unrestricted use, distribution, and reproduction in any medium, provided the original author and source are credited. 